\title{
Magnetically intercalated multilayer silicene
}

\author{
Andrey $M$. Tokmachev ${ }^{1, *}$, Dmitry $V$. Averyanov ${ }^{1}$, Igor A. Karateev ${ }^{1}$, Oleg E. Parfenov ${ }^{1}$, Oleg A. Kondratev ${ }^{1}$, Alexander N. \\ Taldenkov ${ }^{1}$, and Vyacheslav G. Storchak ${ }^{1, *}$ \\ ${ }^{1}$ National Research Centre "Kurchatov Institute”, Kurchatov Sq. 1, 123182 Moscow, Russia
}

\begin{abstract}
Silicene, a Si-based analogue of graphene, is predicted to exhibit topological electronic phases with exotic properties capable to revolutionize electronics. In particular, the silicene structure is highly advantageous for spintronics. However, lack of synthetic routes to free-standing and magnetically functionalized silicene compounds prevents experimental corroboration of the predictions. Here we synthesize $\mathrm{EuSi}_{2}$, multilayer silicene intercalated with inherently magnetic $\mathrm{Eu}$ atoms, on $\mathrm{SrSi}_{2} / \mathrm{Si}(001)$ templates. The resulting films are formed by crystallites of two mutually orthogonal orientations. The structure is firmly established with electron diffraction, X-ray diffraction and electron microscopy. The compound $\mathrm{EuSi}_{2}$ exhibits non-trivial magnetic and transport properties. The data are compared with those for $\mathrm{EuSi}_{2}$ films grown on $\mathrm{SrSi}_{2} / \mathrm{Si}(111)$ templates.
\end{abstract}

\section{Introduction}

The exceptional rise of graphene to prominence in condensed-matter physics has driven a surge in studies of two-dimensional (2D) materials. Buckled 2D Xene sheets formed by group IVA atoms arranged in a honeycomb lattice are of particular interest to nanotechnology due to diversity and high tunability of their electronic states [1]. The most important member of the family of 2D Xenes is silicene, a Si-based analogue of graphene [2, 3]. Similar to graphene, physical properties of silicene are largely determined by massless Dirac fermions. The difference is that the band gap in silicene is easily tuned by chemical functionalization and electric fields. Despite technological difficulties, silicene is demonstrated as a promising material for field-effect transistors [4]. Electric and exchange fields induce different topological states in silicene [3, 5] leading to unconventional properties including quantum anomalous Hall effect, quantum spin Hall effect and valley polarized quantum Hall effect.

Silicene is thought to be especially valuable for application in spintronics [3]. Unlike graphene, silicene benefits from relatively strong spin-orbit interaction, large spin-coherence length and spin-diffusion time. Especially useful are valley-spin coupling and accessible topological states with non-trivial spin transport. However, to make use of these functionalities magnetic states in silicene should be induced, for example by chemical modification. In this respect, adsorption or intercalation of metal atoms are considered as a prospective way to bring diverse magnetic properties into the silicene physics [3].

A lot of predictions are made about unique and exotic properties of silicene compounds but their experimental realization is well behind the theory and electronic structure calculations. The problem is in synthesis of silicene and its derivatives. Synthesis of 2D materials is usually tricky [6] but in the case of silicene it is vastly aggravated by its high chemical reactivity caused by significant buckling of silicene layers. Free-standing silicene is yet to be produced. 2D silicene structures are formed by adsorption of Si on metal substrates; however, a strong hybridization of silicene with the substrate destroys the Dirac states $[2,3,7]$.

It is suggested that intercalation of silicene with active atoms restores Dirac cones [7]. This hypothesis is experimentally corroborated in multilayer silicene intercalated with $\mathrm{Ca}$, a polymorph of $\mathrm{CaSi}_{2}$ [8]. Recently we succeeded in synthesis of Sr-intercalated multilayer silicene, a new polymorph of $\mathrm{SrSi}_{2}$ [9]. It is engineered by epitaxial stabilization on $\mathrm{Si}(111)$ and $\mathrm{Si}(001)$. In contrast to $\mathrm{CaSi}_{2}$, layered $\mathrm{SrSi}_{2}$ enjoys a regular stacking of silicene layers, thus avoiding the momentum shift of Dirac cones from high-symmetry points [9].

Both $\mathrm{CaSi}_{2}$ and $\mathrm{SrSi}_{2}$ are nonmagnetic. To induce magnetic properties into silicene lattice we proposed a synthesis of multilayer silicene intercalated with inherently magnetic atoms [10]. We chose Eu because it is the most active among magnetic metals, the half-filled $f$-shell of $\mathrm{Eu}^{2+}$ provides strong magnetic properties, and the isomorphism of $\mathrm{Eu}(\mathrm{II})$ and $\mathrm{Sr}$ compounds provides an opportunity for successful synthesis. In Ref. [10] we thoroughly explored synthesis and properties of EuSi2 grown on $\mathrm{SrSi}_{2} / \mathrm{Si}(111)$ with silicene layers parallel to the surface. However, many unique properties of silicene lattice come from electric fields acting normal to silicene surface $[3,5]$. Here, we explore transport across magnetic silicene layers by studying in detail $\mathrm{EuSi}_{2}$ synthesized on $\mathrm{Si}(001)$ with a $\mathrm{SrSi}_{2}$ template layer. 


\section{Methods}

The films of silicides are grown by molecular beam epitaxy in the Riber Compact 12 system. The UHV system consists of Gamma Vacuum Titan Ion Pump, Cryo-Torr 8 cryopump, cryopanels and a titanium sublimation pump. The base pressure in the growth chamber is less than $10^{-10}$ Torr. The metals ( $\mathrm{Sr}$ and $\mathrm{Eu}$ ) of $4 \mathrm{~N}$ quality are effused from Knudsen cell sources. The intensity of molecular beams is measured with a Bayard-Alpert ionization gauge. The substrate temperature is determined with a PhotriX MLAAPX/090 infrared pyrometer. The surface is controlled in situ with a RHEED diffractometer.

X-ray diffraction (XRD) study of the films employs Rigaku SmartLab diffractometer with a $9 \mathrm{~kW}$ copper anode. Electron microscopy studies are carried out for cross-sections prepared in Helios scanning electron microscope/focused ion beam (FIB) dual system. The surface of the samples is covered by a layer of Pt. $\mathrm{Ga}^{+}$ ions are used for FIB milling, thinning and cleaning cross-sections. The specimens are protected from oxidation by carbon deposition. The structure of the films is studied with a Titan 80-300 microscope.

Magnetic properties of $\mathrm{EuSi}_{2}$ are studied with MPMS XL-7 Superconducting Quantum Interference Device employing the reciprocating sample option. Transport properties of the films are determined in the van der Pauw configuration with a Lake Shore 9709A measurement system. Ohmic contacts to metallic films are produced by placing a Ga-Sn-Ag alloy.

\section{Results}

Multilayer silicene intercalated with Eu atoms (trigonal $\mathrm{EuSi}_{2}$ ) is less stable than the tetragonal $\mathrm{EuSi}_{2}$. The latter is formed in the reaction of $\mathrm{Eu}$ atoms with $\mathrm{Si}$ surface, irrespective of its orientation [11]. We employ epitaxial stabilization to produce trigonal $\mathrm{EuSi}_{2}$. The first step is to prepare a template of layered $\mathrm{SrSi}_{2}$ on a clean $\mathrm{Si}(001)$ surface following the recipe proposed in Ref. [9]. The synthesis goes through a number of surface reconstructions of $\mathrm{Sr}$ on $\mathrm{Si}(001)$ [12]. Due to natural incommensurability of the hexagonal lattice of $\mathrm{SrSi}_{2}$ and the cubic lattice of $\mathrm{Si}(001)$, the resulting film is formed by crystallites of two orientations differing by lateral rotations by $90^{\circ}$. Silicene layers are orthogonal to the $\mathrm{Si}(001)$ surface for both orientations.

$\mathrm{EuSi}_{2}$ is then grown by directing a beam of Eu atoms $\left(10^{-8}\right.$ Torr) on top of the $\mathrm{SrSi}_{2} / \mathrm{Si}(001)$ template kept at $450{ }^{\circ} \mathrm{C}$. Si atoms go through $\mathrm{SrSi}_{2}$ via the well-known vacancy mechanism. The structure is capped with $\mathrm{SiO}_{\mathrm{x}}$ to prevent its degradation by atmosphere. RHEED images (Fig. 1a) show that the film is formed by two types of $\mathrm{EuSi}_{2}$ crystallites with orthogonal orientations repeating the morphology of the underlying $\mathrm{SrSi}_{2}$. Regretfully, the epitaxial stabilization is short-range: the film adopts the most stable form of $\mathrm{EuSi}_{2}$ [11] when the thickness of $\mathrm{EuSi}_{2}$ exceeds $7 \mathrm{~nm}$. It becomes evident in RHEED images (Fig. 1b) revealing appearance of the pattern corresponding to tetragonal $\mathrm{EuSi}_{2}$.
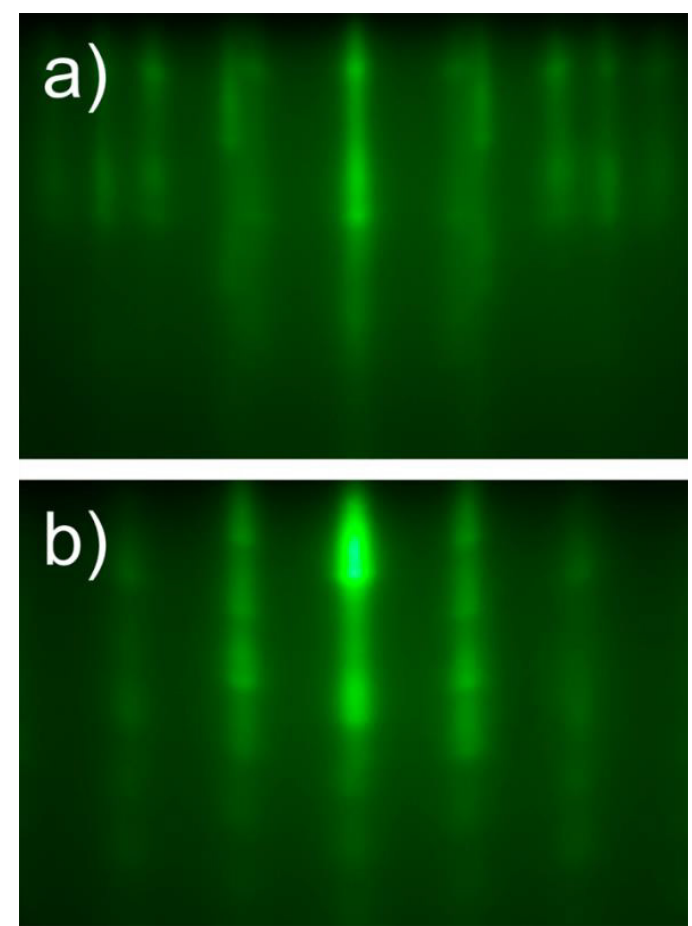

Fig. 1. RHEED image of $\mathrm{EuSi}_{2}$ grown on $\mathrm{SrSi}_{2} / \mathrm{Si}(001)$ viewed along azimuth [110] of the substrate. a) thickness less than $7 \mathrm{~nm}$; b) thickness exceeds $7 \mathrm{~nm}$.

The structure of the films is further characterized ex situ. XRD $\theta-2 \theta$ scan for the $\mathrm{SiO}_{\mathrm{X}} / \mathrm{EuSi}_{2} / \mathrm{SrSi}_{2} / \mathrm{Si}(001)$ film is shown in Fig. 2. Although peaks from $\mathrm{SrSi}_{2}$ and $\mathrm{EuSi}_{2}$ are not resolved due to isomorphism of the compounds and closeness of the corresponding lattice constants, the scan is consistent with the proposed orientation of crystallites and the absence of any alien phases.

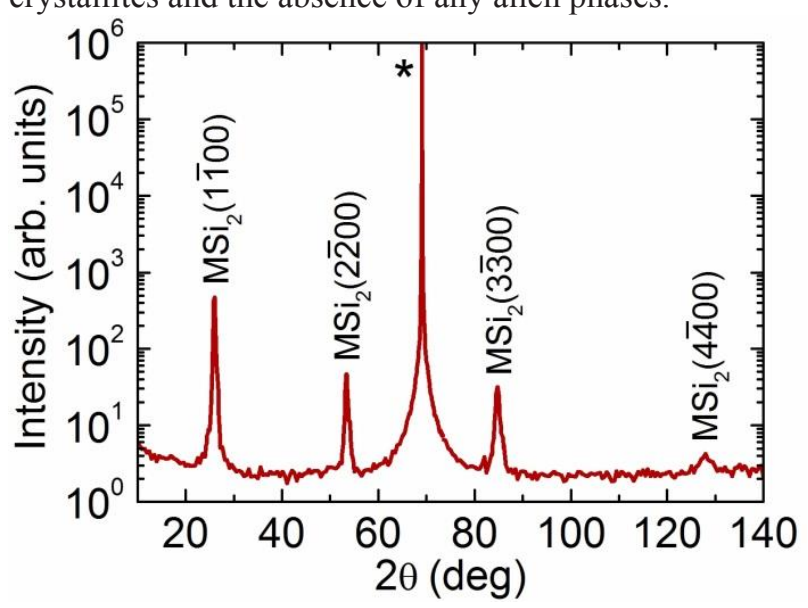

Fig. 2. $\theta-2 \theta$ XRD scan for the $\mathrm{SiO}_{\mathrm{x}} / \mathrm{EuSi}_{2} / \mathrm{SrSi}_{2} / \mathrm{Si}(001)$ film. Peaks from $\mathrm{SrSi} 2$ and $\mathrm{EuSi} 2$ are not resolved, a peak from $\mathrm{Si}$ is marked *.

The atomic structure of the films is revealed by electron microscopy. Fig. 3 shows HAADF-STEM image for one of the crystallites with silicene layers running normal to the cross-section. The image confirms the orientational relation between $\mathrm{SrSi}_{2}$ and $\mathrm{EuSi}_{2}$. It also reveals the presence of edge dislocations: extra halfplanes of atoms are introduced at the $\mathrm{EuSi}_{2} / \mathrm{SrSi}_{2}$ boundary to account for some mismatch between the lattices. Fig. 4 shows a crystallite of the other 
orientation, with silicene layers parallel to the crosssection. We employ the bright-field mode with reduced Z-contrast to show the honeycomb structure of silicene. The boundary between $\mathrm{EuSi}_{2}$ and $\mathrm{SrSi}_{2}$ is formed by a single layer of mixed $\mathrm{Eu}_{\mathrm{x}} \mathrm{Sr}_{1-\mathrm{x}} \mathrm{Si}_{2}$ silicide marked as red rectangle.

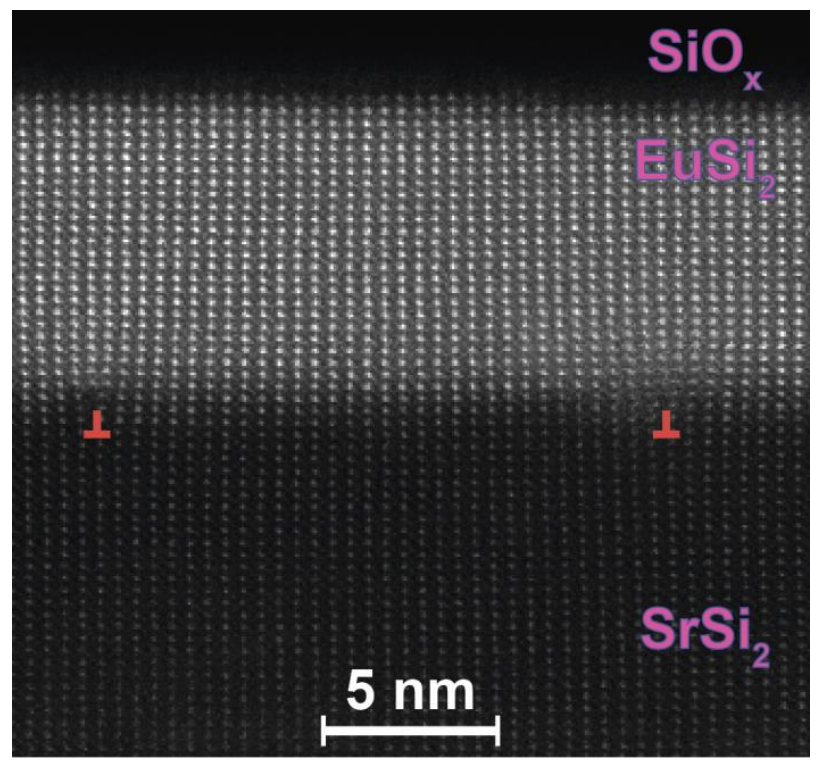

Fig. 3. Cross-sectional HAADF-STEM image of the $\mathrm{SiO}_{\mathrm{x}} / \mathrm{EuSi}_{2} / \mathrm{SrSi}_{2} / \mathrm{Si}(001)$ film. Edge dislocations in $\mathrm{EuSi}_{2}$ are marked by red pointers.

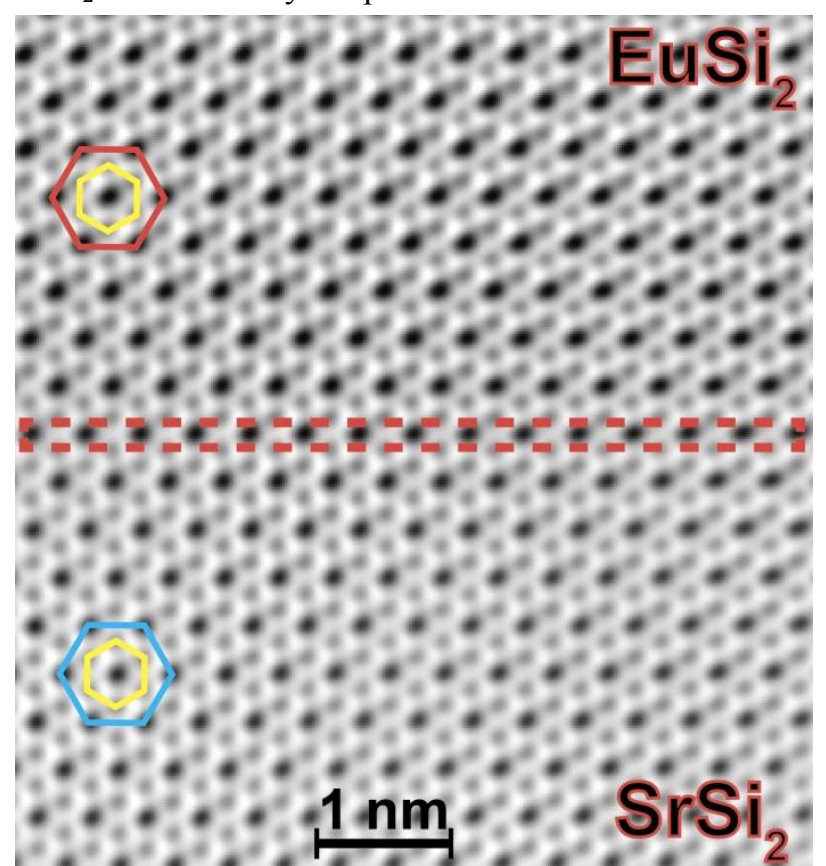

Fig. 4. Cross-sectional BF-STEM image of the $\mathrm{SiO}_{\mathrm{x}} / \mathrm{EuSi}_{2} / \mathrm{SrSi}_{2} / \mathrm{Si}(001)$ film. It shows the boundary between $\mathrm{EuSi}_{2}$ and $\mathrm{SrSi}_{2}$ phases as well as hexagons of $\mathrm{Eu}$ (red), $\mathrm{Sr}$ (blue) and $\mathrm{Si}$ (yellow).

The aim of making trigonal $\mathrm{EuSi}_{2}$ is to get a magnetic (spin) response from a silicene-based system. Fig. 5 shows temperature dependence of the magnetic moment measured in magnetic field of $1 \mathrm{~T}$ directed along 3 axes. The film demonstrates antiferromagnetic (AFM) response with moments oriented normal to the plane and parallel to silicene layers.

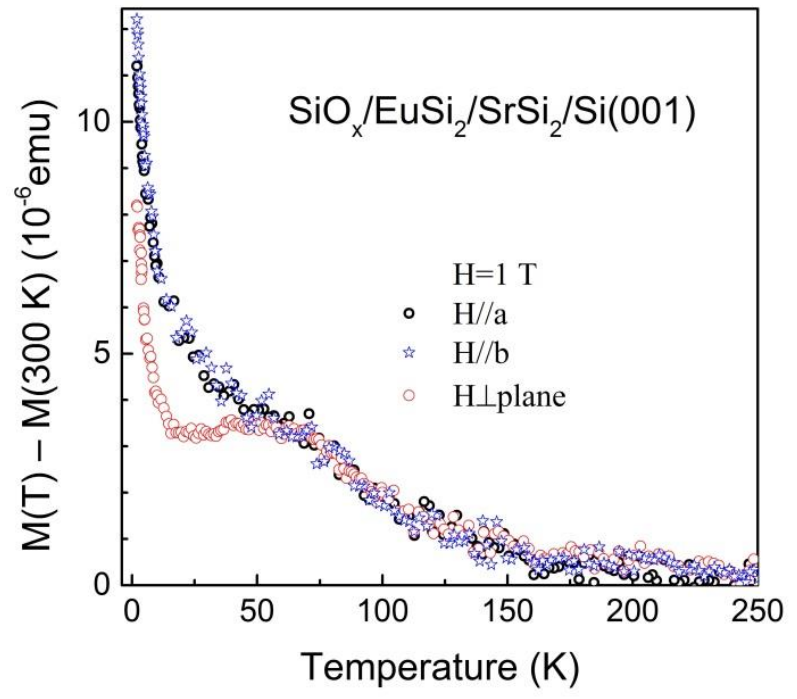

Fig. 5. Temperature dependence of magnetic moment of the $\mathrm{SiO}_{\mathrm{x}} / \mathrm{EuSi}_{2} / \mathrm{SrSi}_{2} / \mathrm{Si}(001)$ film for different directions of the external field $(1 \mathrm{~T})$ with respect to the substrate: (001) - red, (110) - black and (110) - blue.
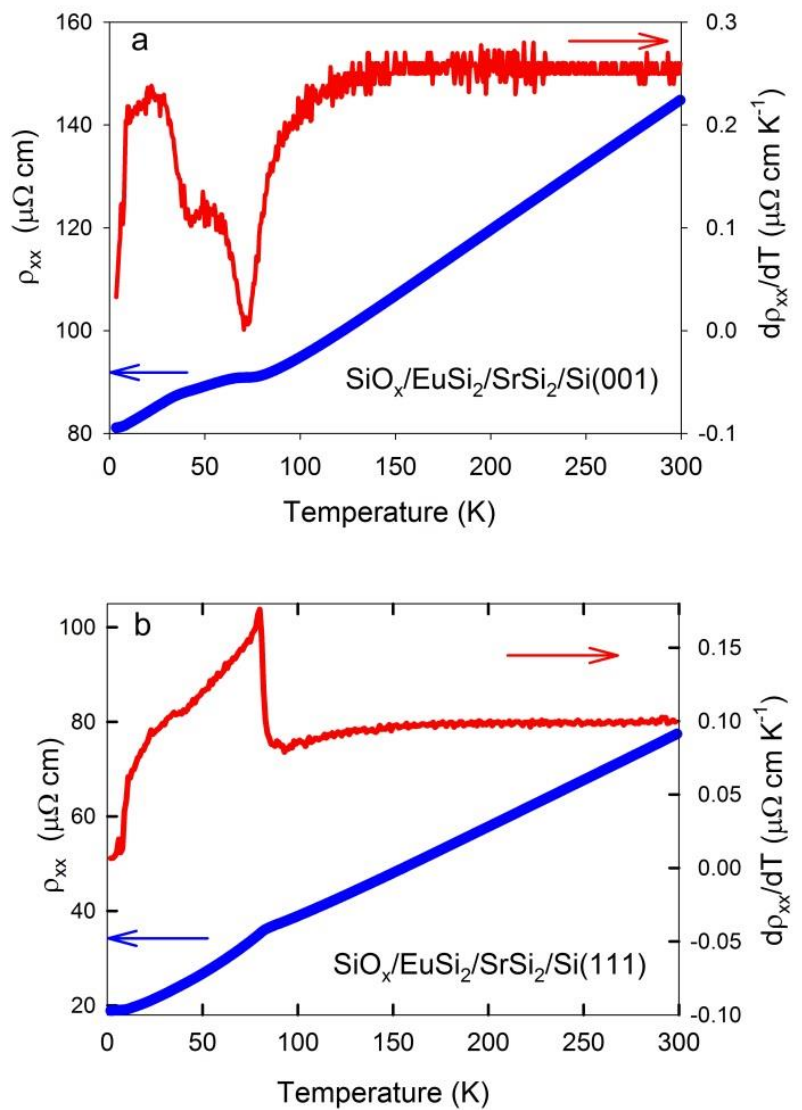

Fig. 6. Temperature dependence of resistivity $\rho_{x x}$ (blue) and its temperature derivative $d \rho_{x x} / d T$ (red) for thin films: a) $\mathrm{SiO}_{\mathrm{x}} / \mathrm{EuSi}_{2} / \mathrm{SrSi}_{2} / \mathrm{Si}(001)$ and b) $\mathrm{SiO}_{\mathrm{x}} / \mathrm{EuSi}_{2} / \mathrm{SrSi}_{2} / \mathrm{Si}(111)$.

Thus, we succeeded in inducing magnetism into a system based on silicene layers. It is reasonable to expect that the proximity of magnetic $\mathrm{Eu}^{2+}$ ions can result in non-trivial transport properties of the films, especially for the transport across a stack of silicene layers. Temperature dependence of resistivity $\rho_{x x}$ and its 
temperature derivative for $\mathrm{EuSi}_{2}$ grown on $\mathrm{Si}(001)$ and $\mathrm{Si}(111)$ are shown in Fig. 6. In the latter case the transport is carried out along silicene layers, while in the former case carriers move partly along the layered structure and partly across it. Naturally, $\mathrm{SrSi}_{2}$ also contributes into the film conductivity but this transport channel shows no anomalies [9]. The resistivity of a magnetic metal is dominated by local spin fluctuations; the temperature dependence of $d \rho_{x x} / d T$ should follow that of the magnetic specific heat. The opposite sign of derivative changes at the AFM transition for the $\mathrm{EuSi}_{2} / \mathrm{SrSi}_{2} / \mathrm{Si}(001)$ and $\mathrm{EuSi}_{2} / \mathrm{SrSi}_{2} / \mathrm{Si}(111)$ structures is a consequence of different direction of magnetic moment with respect to the current in the two films.

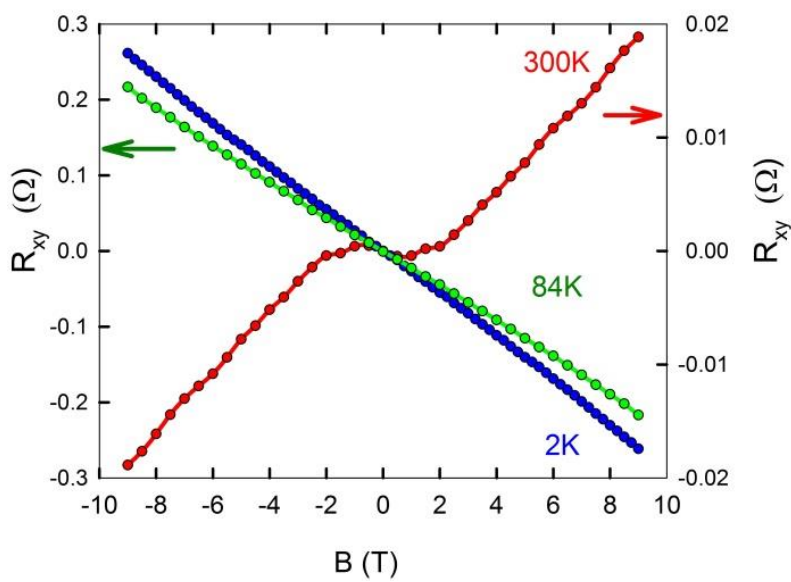

Fig. 7. Magnetic field dependence of Hall resistance $R_{x y}$ for $\mathrm{SiO}_{\mathrm{x}} / \mathrm{EuSi}_{2} / \mathrm{SrSi}_{2} / \mathrm{Si}(001)$ structure measured at three temperatures: $2 \mathrm{~K}$ (blue), $84 \mathrm{~K}$ (green) and $300 \mathrm{~K}$ (red).

In Ref. [10] we found an unconventional magnetoresistance and its temperature dependence in transport across silicene layers. Here, we study Hall resistance $R_{x y}$ for the films. In the case of the $\mathrm{EuSi}_{2} / \mathrm{SrSi}_{2} / \mathrm{Si}(111)$ structure, Hall response is unexceptional - dependence of $R_{x y}$ on $B$ is linear at all $T$; the sign of the derivative corresponds to electron carriers. The situation is quite different for the $\mathrm{EuSi}_{2} / \mathrm{SrSi}_{2} / \mathrm{Si}(001)$ structure, as shown in Fig. 7. The behaviour of the Hall resistance at low temperatures (including those above $T_{\mathrm{N}}$ ) is quite similar to that of $\mathrm{EuSi}_{2}$ on $\mathrm{Si}(111)$. However, the sign of the Hall effect changes from electron-like to hole-like as $\mathrm{T}$ approaches room temperature. More importantly, this change in the electronic structure is accompanied by anomalous contribution to the Hall resistivity, which is highly unusual for a paramagnet. This behaviour may be caused by interplay of more than one band.

\section{Conclusions}

Silicene is one of the most promising materials for nanoelectronics. Its structure ensures efficient transport properties which can be manipulated by external stimuli. A major promise of silicene is for spin-dependent phenomena and transport. However, the bottleneck of silicene studies - synthesis - precludes the theoretical predictions from experimental verification. Here, we attempted to bridge the gap between the theory and experiment by studies of stacks of silicene layers intercalated with magnetic Eu atoms. We described the complex synthesis procedure and verified the atomic structure of the resulting layered polymorph of $\mathrm{EuSi}_{2}$ grown on $\mathrm{Si}(001)$ with a $\mathrm{SrSi}_{2}$ template. The film exhibits a complex magnetic structure dominated by antiferromagnetism. This conclusion is confirmed by studies of transport across silicene layers. The electronic structure of the films changes as temperature goes up from $2 \mathrm{~K}$ to $300 \mathrm{~K}$, signified by changes in the carrier type and unexpected room-temperature anomaly in Hall measurements. The results call for additional studies, both experimental and theoretical.

This work is partially supported by NRC "Kurchatov Institute", the Russian Foundation for Basic Research (grants 16-0700204, 16-29-03027, and 17-07-00170), and the Russian Science Foundation (grant 14-19-00662). The measurements are partially carried out using the equipment of the resource centres of electrophysical, laboratory X-ray, and electron microscopy techniques of NRC "Kurchatov Institute".

\section{References}

1. A. Molle, J. Goldberger, M. Houssa, Y. Xu, S.-C. Zhang, D. Akinwande, Nature Mat. 16, 163 (2017).

2. C. Grazianetti, E. Cinquanta, A. Molle, 2D Mat. 3, 012001 (2016).

3. J. Zhao, H. Liu, Z. Yu, R. Quhe, S. Zhou, Y. Wang, C.C. Liu, H. Zhong, N. Han, J. Lu, Y. Yao, K. Wu, Prog. Mat. Sci. 83, 24 (2016).

4. L. Tao, E. Cinquanta, D. Chiappe, C. Grazianetti, M. Fanciulli, M. Dubey, A. Molle, D. Akinwande, Nature Nanotech. 10, 227 (2015).

5. M. Ezawa, Phys. Rev. Lett. 109, 055502 (2012).

6. A.J. Mannix, B. Kiraly, M.C. Hersam, N.P. Guisinger, Nature Rev. Chem. 1, 0014 (2017).

7. R. Quhe, Y. Yuan, J. Zheng, Y. Wang, Z. Ni, J. Shi, D. Yu, J. Yang, J. Lu, Sci. Rep. 4, 5476 (2014).

8. E. Noguchi, K. Sugawara, R. Yaokawa, T. Hitosugi, H. Nakano, T. Takahashi, Adv. Mat. 27, 856 (2015).

9. A.M. Tokmachev, D.V. Averyanov, I.A. Karateev, O.E. Parfenov, A.L. Vasiliev, S.N. Yakunin, V.G. Storchak, Nanoscale 8, 16229 (2016).

10. A.M. Tokmachev, D.V. Averyanov, I.A. Karateev, O.E. Parfenov, O.A. Kondratev, A.N. Taldenkov, V.G. Storchak, Adv. Funct. Mat. 27, 1606603 (2017).

11. D.V. Averyanov, A.M. Tokmachev, C.G. Karateeva, I.A. Karateev, E.F. Lobanovich, G.V. Prutskov, O.E. Parfenov, A.N. Taldenkov, A.L. Vasiliev, V.G. Storchak, Sci. Rep. 6, 25980 (2016).

12. D.V. Averyanov, C.G. Karateeva, I.A. Karateev, A.M. Tokmachev, M.V. Kuzmin, P. Laukkanen, A.L. Vasiliev, V.G. Storchak, Mater. \& Design 116, 616 (2017). 